DOI 10.15593/2409-5125/2016.04.04

УДК $697.952: 692.492$

\title{
T.C. Крашенинникова
}

Университет Ноттингема (Великобритания)

\author{
А.В. Гришкова \\ Пермский национальный исследовательский \\ политехнический университет
}

\section{АНАЛИЗ ПАРАМЕТРОВ МИКРОКЛИМАТА В ЗДАНИИ С АТРИУМОМ В ЗАДАННЫХ КЛИМАТИЧЕСКИХ УСЛОВИЯХ С ИСПОЛЬЗОВАНИЕМ ПРОГРАММЫ ANSYS FLUENT}

При проектировании и строительстве современных зданий одними из важнейших критериев сегодня становятся энергоэффективность и экологическая безопасность. Только применение инженерных и архитектурных решений в комбинации способно обеспечить достижение высоких показателей для здания по данным критериям. Одним из примеров такого подхода являются здания с атриумами. Применение атриума может снизить потребление электрической энергии за счет естественного освещения и сократить теплопотери ограждающими конструкциями. В то же время атриум увеличивает естественный напор воздуха, позволяющий использовать вместо энергозатратных механических систем вентиляции более экономичные естественные системы.

В статье приведен анализ использования программного продукта ANSYS FLUENT при моделировании работы естественной вентиляции для здания с атриумом. В качестве методики исследования параметров микроклимата использован принцип, применяющийся исследователями в Великобритании. Произведена оценка полученных результатов и их анализ на основании полученного графического материала. Объектом исследования послужило офисное здание с атриумом; верх атриума расположен на 6 м выше уровня крыши. Для расчета выбран летний период года и температура наружного воздуха $23,4{ }^{\circ} \mathrm{C}$. Приведены результаты определения количества приточного и вытяжного воздуха, а также скорости воздуха в приточных и вытяжном отверстиях. Сделан вывод о необходимости проведения подобных исследований для естественных систем вентиляции и режимов их работы с моделированием и оценкой эффективности разрабатываемых вариантов систем.

Ключевые слова: естественная вентиляция, вентиляция зданий с атриумом, программный продукт ANSYS FLUENT, метод конечных объемов. 
Вопросы энергоэффективности, энергосбережения и снижения нагрузки на окружающую среду уже продолжительное время ставятся во главу угла при проектировании и строительстве современных зданий во всем мире. На основании общеевропейских директив по сокращению энергопотребления зданий и снижения экологической нагрузки в государствах Евросоюза, так же как и в других странах мира, разрабатываются свои национальные стандарты и методологии определения основных энергетических и экологических характеристик зданий и сооружений на их соответствие современным требованиям [1]. Наряду с национальными стандартами все более широкое применение получают также независимые рейтинговые системы оценки экологической и энергетической устойчивости зданий, призванные повысить престиж энергоэффективных зданий, сертифицируя их по балльной системе. На сегодняшний день наиболее известными и популярными являются системы BREEM (Великобритания), LEED (CША), Green Star (Австралия), Estidama (Ближний Восток). Энергоэффективные и экологически устойчивые здания, имеющие подобные сертификаты наряду с понижением эксплуатационных затрат и более высоким уровнем комфорта помещений, как правило, имеют высокую маркетинговую привлекательность для инвесторов, девелоперов, проектировщиков и собственников, являясь современным и высококачественным продуктом, обеспечивающим его производителю/поставщику конкурентные преимущества на рынке «зеленого строительства». В России начата работа по созданию собственных национальных стандартов в «зеленом» строительстве и по оценке энергоэффективности зданий. Первым таким стандартом стал ГОСТ Р 54964-2012 «Оценка соответствия. Экологические требования к объектам недвижимости». Наряду с государственными стандартами существует и отечественная рейтинговая система Green Zoom.

Базовый подход, лежащий в основе всех вышеперечисленных систем и стандартов, - это снижение энергопотребления здания путем введения некоторых экологически и энергетически устойчивых требований к процессу его проектирования и строительства. Зачастую выполнение данных требований может быть обеспечено путем комбинации инженерных решений с архитек- 
турными. Например, достижение высокого уровня комфорта в здании может быть достигнуто его планировкой с учетом естественного распределения температурных и скоростных полей внутри здания. Для осуществления этой стратегии применяется математическое моделирование (CFD), позволяющее подтвердить расчетами заявленный в здании (помещении) уровень комфорта.

Присутствие на рынке программных пакетов по моделированию скоростных и температурных полей способствует все большему распространению новых архитектурных решений, призванных повысить энергоэффективность и экологическую безопасность. Одним из таких решений являются здания с атриумами и светопрозрачными куполами [2]. Атриум является не только частью архитектурной концепции здания, но также может решать целый ряд инженерных задач. Так, использование естественного освещения в зданиях с атриумами позволяет не только экономить электрическую энергию, но и сократить теплопотери [3]. Преимущества атриума можно использовать и для повышения энергоэффективности систем вентиляции и определения целесообразного потокораспределения вентиляционного воздуха.

Как правило, здания с атриумом - это общественные здания, где требуемый воздухообмен гораздо выше, чем в жилых. Часто в общественных зданиях только системы механической вентиляции способны обеспечить требуемые параметры микроклимата. Наличие же атриума позволяет увеличить естественный напор, обеспечив условия для работы естественной вентиляции, которая не требует мест для размещения приточных камер и вентиляционных каналов. В свою очередь, это решение позволяет частично или полностью отказаться от механических систем, потребляющих энергию, выработанную путем сжигания органического топлива с выбросами парниковых газов в атмосферу.

Однако использование естественных систем вентиляции связано с ограничением регулирования производительности и сложностью распределения приточного наружного воздуха, а следовательно, с проблемой ликвидации неточностей, допускаемых при проектировании. Поэтому перед внедрением конкретные проектные решения должны проходить тщательную проверку и тестирование. 
В тех случаях, когда прямое аналитическое решение задачи затруднено, а на экспериментальное исследование требуются значительные средства, исследователи и инженеры при решении технических проблем применяют компьютерное математическое моделирование [4-6]. В частности, ANSYS FLUENT - это один из основных продуктов, предлагаемый компанией ANSYS Inc. для решения задач гидрогазодинамики общего назначения [7].

Компьютерное моделирование можно использовать при проектировании вентиляции для определения параметров воздуха в определенной точке в заданное время для конкретных проектных решений, например при максимальной нагрузке или для типичных летних или зимних условий [8, 9]. В процессе проектирования этот способ позволяет проверить проектное решение и протестировать потенциально проблемные зоны помещения на соответствие требуемым параметрам или заявленному уровню комфорта.

Целью исследования явился анализ результатов моделирования параметров воздуха для зданий с атриумом при естественной вентиляции. Моделирование произведено с помощью программного пакета ANSYS FLUENT по методике, используемой в университетах Великобритании. Оценка полученных визуальных результатов включает в себя рекомендации по техническим решениям для вентиляции рассматриваемого здания.

Для достижения поставленной цели выполнен анализ микроклимата в здании с атриумом в заданных климатических условиях, включая оценку параметров в критических областях.

Таким образом, при оценке результатов моделирования должны быть получены ответы на следующие практические вопросы:

1. Будет ли естественная вентиляция работать в предложенном здании, с учетом его назначения, его формы в предложенных условиях?

2. Какие размеры отверстий должны быть приняты для естественной вентиляции в здании с учетом его назначения в предложенных условиях?

Для моделирования работы системы естественной вентиляции было выбрано трехэтажное здание с атриумом. Здание имеет открытые офисные пространства, на каждом этаже объединенные в атриум. Верх атриума расположен на 6 м выше крыши здания. 
Перекрытия толщиной 0,3 м, остекление - 0,1 м. Здание запроектировано с естественной вентиляцией, работающей за счет разности давлений внутреннего и наружного воздуха. Размер оконных проемов в наружной стене увеличивается с первого до третьего этажа и составляет соответственно 0,$3 ; 0,4$ и 0,5 м. Размер отверстий во внутренних перегородках 0,5 м. Температура наружного воздуха в дневное время для моделирования принята произвольно $23,4{ }^{\circ} \mathrm{C}$. Эквивалентные внутренние тепловые нагрузки с площади пола офисов составляют $43 \mathrm{Bт} / \mathrm{m}^{2}$. Теплопоступления от солнечной радиации на поверхность наружной стены атриума и его покрытие составляют 44 и $40 \mathrm{Bт} / \mathrm{M}^{2}$ соответственно. Указанные теплопоступления представляют тепловые потоки только от конвективного теплообмена. Теплообменом за счет теплопроводности и радиации при моделировании можно пренебречь.

Размеры здания и внешний вид представлены на рис. 1.
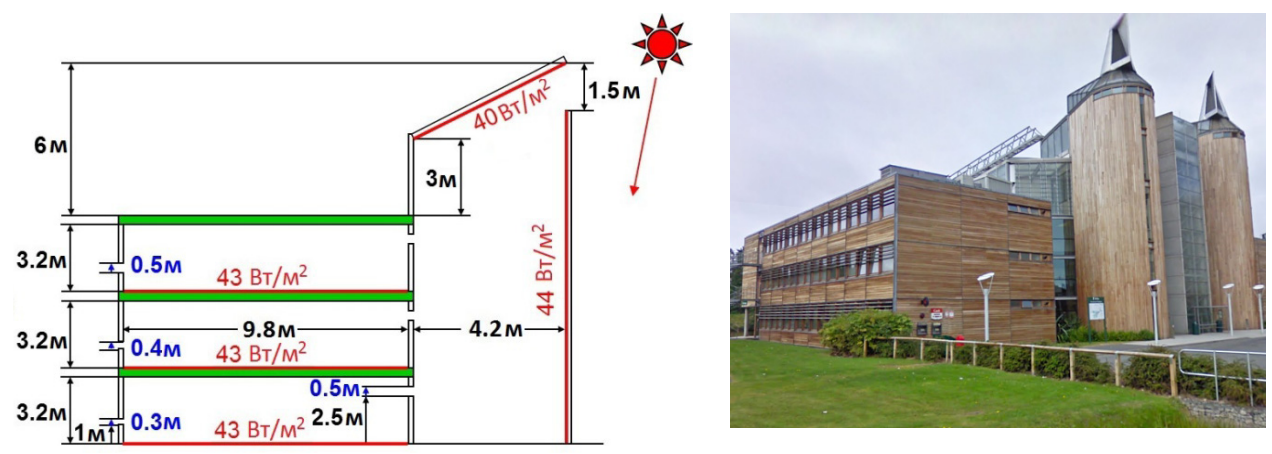

Рис. 1. Здание с атриумом

При подготовке к процессу моделирования в программе ANSYS FLUENT были выполнены следующие операции:

- создание геометрической схемы модели: вычислительного домена;

- деление домена на множество мелких доменов (сетка конечных объемов);

- определение физического процесса движения воздуха, который требуется смоделировать (учитывая, что рассматриваемый поток не является полностью турбулентным);

- определение свойств воздушного потока;

- выбор граничных условий. 
Решение поставленной задачи в ANSYS FLUENT состоит из следующих шагов:

1) выбор стратегии решения;

2) назначение критерия сходимости;

3) назначение числа итераций.

После завершения процесса моделирования результаты были проанализированы для их дальнейшей оценки.

Использованная методика основывается на методе конечных объемов. Следует отметить, что точность решения подобного рода задач во многом зависит от того, насколько корректно произведено деление вычислительного домена на конечные объемы (элементы). При этом не только размер элементов сетки играет роль, но и их форма и ориентация. Плотность сетки должна быть больше в зонах высокой концентрации неодномерных градиентов и в зонах, представляющих особый интерес [10]. Важным параметром является количество элементов в домене. От этого напрямую зависит количество памяти, требуемое для обработки задачи процессором. Для домена определенного размера количество элементов определяет размер сетки (т.е. расстояния, на которых будут рассчитываться неодномерные градиенты), что очень важно для достижения сходимости и точности [1].

Решение уравнений находится как в большинстве итерационных методов путем минимизации невязки. В первом опыте вычислений было проведено 7000 итераций для базовой конечноэлементной сетки. Однако в результате не была достигнута требуемая сходимость результатов вычислений.

При моделировании процессов гидрогазодинамики целесообразно уменьшение шага конечно-элементной сетки вдоль границ критических областей, т.е. вблизи стен. Данная процедура позволяет более корректно учесть возникновение значительных градиентов скоростей воздуха в пристеночной области при взаимодействии воздушных потоков.

После применения функции «сгущения сетки в пристеночных областях» рассматриваемого здания было проведено дополнительно еще 3000 итераций. При этом требуемая сходимость результатов вновь не была достигнута и потребовалось дополнительное уменьшение шага конечно-элементной сетки. Было применено так назы- 
ваемое «местное сгущение сетки» [11]. Данная функция измельчает сетку внутри или снаружи определенной зоны, в данном случае внутри всего пространства рассматриваемого здания.

После дополнительных 3000 итераций требуемая сходимость результатов была достигнута.

При моделировании оценивались ключевые параметры микроклимата помещений здания: поля распределения скоростей и температур. Данные параметры оказывают большое влияние на создание комфорта и особенно важны в помещениях с естественной вентиляцией. Скорость потока напрямую влияет на распределение концентраций загрязняющих веществ от источника загрязнения во времени. Температурное поле оказывает влияние на тепловые потоки через оболочку здания, в силу результирующего распределения гидростатического давления [12].

На рис. 2 представлено графическое распределение поля скоростей воздуха внутри здания для базовой конечно-элементной сетки и общего числа итераций 7000. Общая модель распределения скоростей воздушного потока в здании представлена достаточно корректно. Поток воздуха проходит через приточные отверстия, через пространства этажей, аккумулируется в атриуме и наконец выбрасывается наружу через вытяжное отверстие. Также можно видеть, что скорости воздушного потока варьируются в разных зонах. Тем не менее значения скоростей недостаточно точны для того, чтобы оценить микроклимат каждого помещения, так как в данном случае результаты решения не достигли требуемой сходимости.

На рис. 3 показано графическое распределение поля скоростей после применения уменьшения шага сетки вдоль границ и последующих 3000 итераций. Как можно видеть, общая модель распределения воздушного потока в здании остается такой же, но с более детальным представлением картины распределения скоростей в приграничных зонах. В свою очередь в пространстве атриума можно наблюдать очертания кольцевых вихрей, которых практически не было видно при применении только базовой конечно-элементной сетки. Также видно, что диапазон изменения скоростей воздушного потока стал несколько шире благодаря более точному вычислению и представлению значительных градиентов скорости в пристенных областях. 


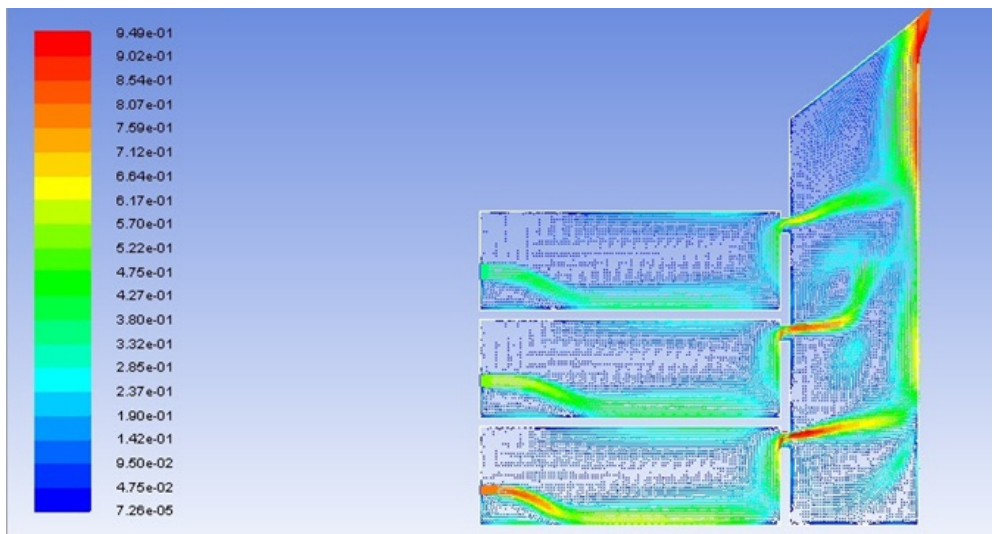

Рис. 2. Поля распределения скорости воздушных потоков (м/с) при 7000 итерациях (опыт 1)

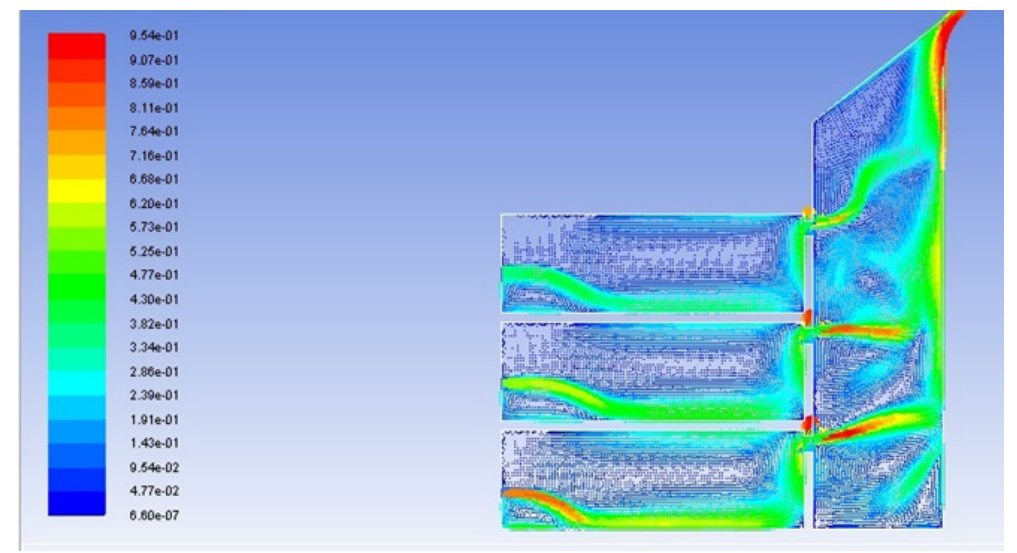

Рис. 3. Поля распределения скорости воздушных потоков (м/с) при уменьшении шага сетки вдоль границ и дополнительных 3000 итераций (опыт 2)

Графическое изображение поля скоростей после применения местного сгущения сетки и дальнейших 3000 итераций представлено на рис. 4. Видно, что общая модель распределения воздушного потока в здании осталась неизменной, но с более детальным представлением (кольцевые вихри в атриуме просматриваются отчетливо), а диапазон изменения скоростей воздушного потока вновь стал шире благодаря более точным расчетам.

Результаты распределения скоростей воздушного потока внутри рассматриваемого здания показывают, что приточный воздух поступает непосредственно в рабочую зону (предполагая, что люди в офисном здании работают в сидячем положении), вы- 
тесняя собой загрязненный воздух. На каждом этаже скорость возрастает в районе пола и до верха приточного отверстия (около $5 \mathrm{~m} / \mathrm{c})$, в то время как в остальном пространстве помещений скорость около $0,1 \mathrm{~m} / \mathrm{c}$.

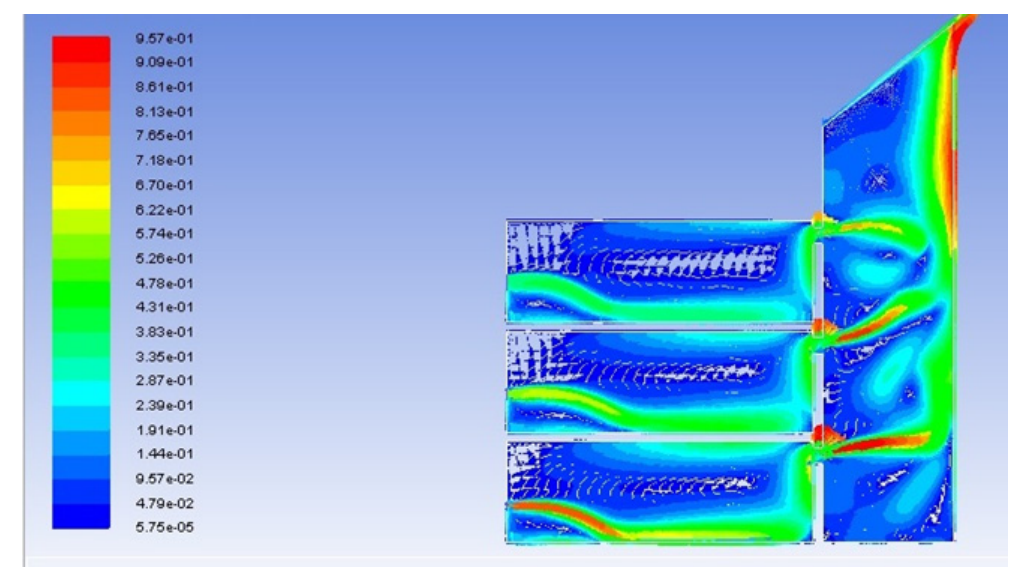

Рис. 4. Поля распределения скорости воздушных потоков (м/с) после применения местного сгущения сетки и дальнейших 3000 итераций (опыт 3)

Движение воздуха более интенсивно на первом этаже (скорость потока достигает $0,9 \mathrm{~m} / \mathrm{c}$ вблизи приточного отверстия) и менее интенсивно на верхнем этаже (скорость около 0,5 м/с). Такое интенсивное движение воздуха достигается наличием атриума, который благодаря своей высоте обеспечивает увеличение разности давлений внутреннего и наружного воздуха. С другой стороны, интенсивное движение воздуха в рабочей зоне (скорость воздушного потока более $0,25 \mathrm{~m} / \mathrm{c}$ ) способствует понижению температуры в ней. Этот эффект желателен в теплый период года. Если эффект охлаждения нежелателен, то он может вызвать жалобы на сквозняк $[13,14]$. Таким образом, в данном случае можно рекомендовать расположить приточные отверстия ближе к потолку во избежание сквозняков в рабочей зоне.

На рис. 5 представлено графическое изображение температурного поля в градусах Кельвина для базовой сетки конечных элементов и 7000 итераций. Общее распределение температур воздуха внутри рассматриваемого здания представлено достаточно отчетливо. Воздух с наружной температурой проходит через приточные отверстия и охлаждает помещения. Затем воздух, уже 
нагретый внутренними теплопоступлениями, аккумулируется в атриуме и выбрасывается через вытяжное отверстие. Тем не менее данные значения температур определены с недостаточной точностью для оценки микроклимата здания, так как в данном случае в результате решения не достигнута требуемая сходимость.

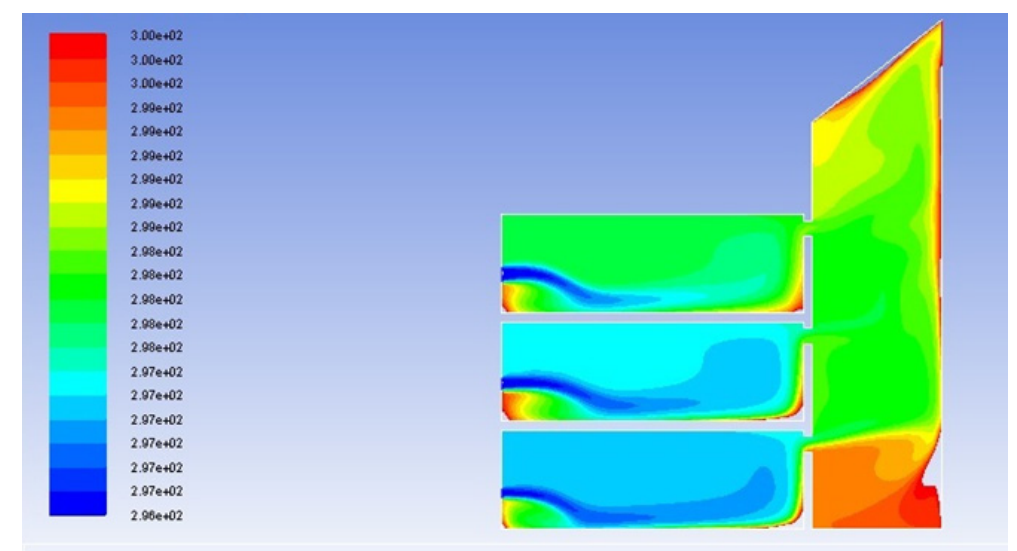

Рис. 5. Графическое представление температурного поля (К).

Базовая сетка конечных элементов (опыт 1)

После применения уменьшения шага сетки вдоль границ и дальнейших 3000 итераций получено графическое изображение температурного поля (рис. 6) с более детальным представлением температур в пристенных областях.

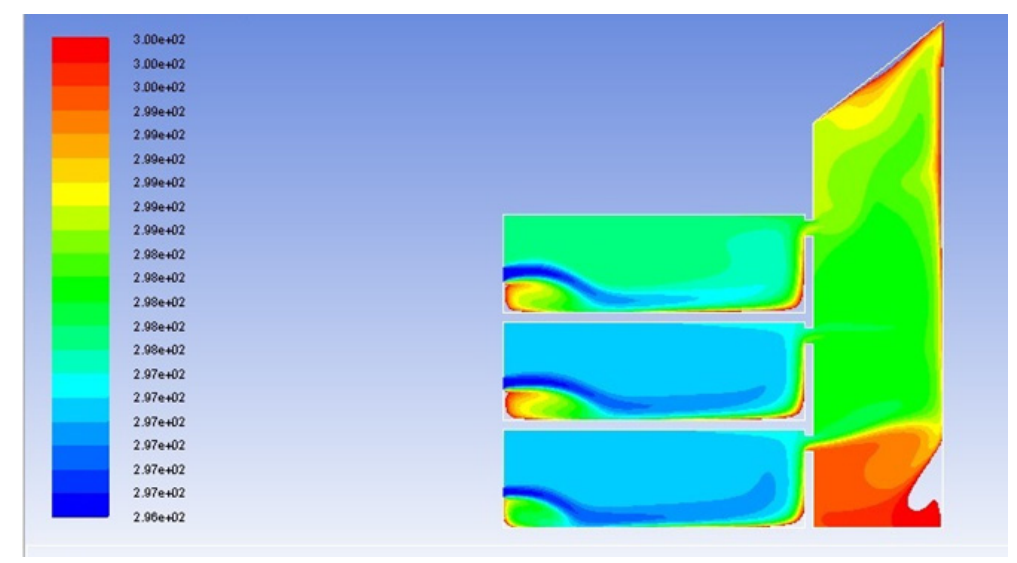

Рис. 6. Графическое представление температурного поля (К) при уменьшении шага сетки вдоль границ (опыт 2) 
После местного сгущения сетки и дополнительных 3000 итерациях общая модель распределения температур в здании остается такой же, но с более детальным представлением (рис. 7). Требуемая сходимость результатов решения достигнута и это позволяет производить дальнейший анализ распределения температур в здании.

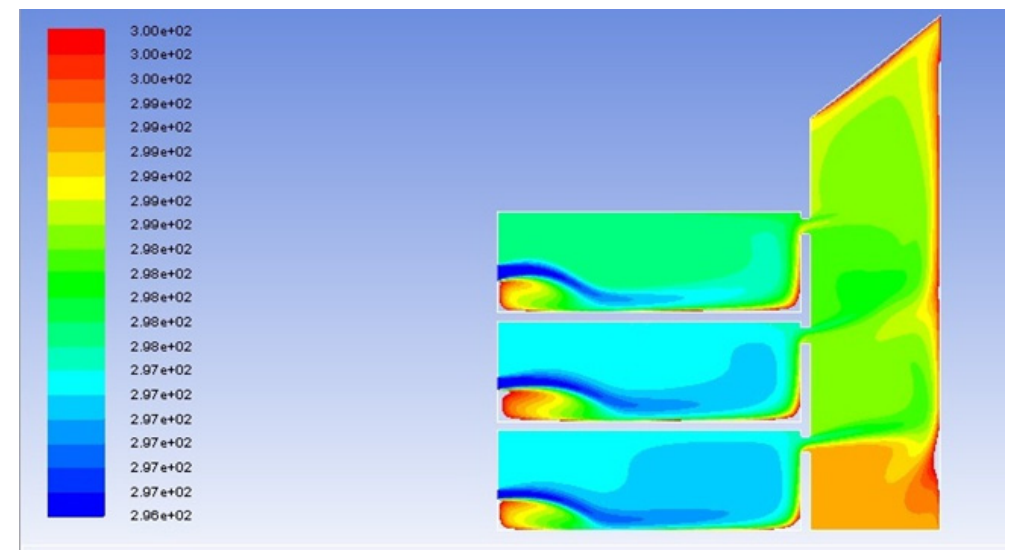

Рис. 7. Графическое представление температурного поля (К) при местном сгущении сетки (опыт 3)

Верхняя граница комфортной температуры для данных условий около $27{ }^{\circ} \mathrm{C}$ [10] (здания с естественной вентиляцией могут считаться комфортными при более широком диапазоне температур внутреннего воздуха, чем здания с кондиционированием воздуха). Распределение температур воздуха показывает, что температура воздуха более низкая на нижнем этаже (около $24{ }^{\circ} \mathrm{C}$ ) и более высокая на верхнем этаже (около $25{ }^{\circ} \mathrm{C}$ в районе потолка и около $24{ }^{\circ} \mathrm{C}$ в районе пола). Наиболее высокие температуры расположены в зоне атриума. Кроме теплопоступлений от удаляемого воздуха атриум также нагревается теплопоступлениями от солнечной радиации через его ограждающие конструкции. Более высокие температуры в зоне вытяжных отверстий обусловливают увеличение гравитационного естественного давления.

Массовый расход воздуха, входящего в здание, должен соответствовать массовому расходу воздуха, покидающего его. По данному критерию можно судить о степени сходимости решения. В табл. 1 представлен отчет о вентиляционных расходах, полученных в результате расчета для базовой сетки конечных элементов 
и 7000 итераций (опыт 1), после применения уменьшения шага сетки вдоль границ и дальнейших 3000 итераций (опыт 2) и после местного сгущения сетки и последующих 3000 итераций (опыт 3).

Таблица 1

Определение вентиляционных расходов воздуха, кг/с

\begin{tabular}{|l|c|c|c|}
\hline \multicolumn{1}{|c|}{ Зона } & Опыт 1 & Опыт 2 & Опыт 3 \\
\hline Приточное отверстие 1 (первый этаж) & 0,277 & 0,274 & 0,285 \\
\hline Приточное отверстие 2 (второй этаж) & 0,267 & 0,259 & 0,259 \\
\hline Приточное отверстие 3 (третий этаж) & 0,210 & 0,203 & 0,201 \\
\hline Вытяжное отверстие & $-0,754$ & $-0,736$ & $-0,745$ \\
\hline Невязка & $-0,0022$ & $-0,0003$ & 5,64 е-05 \\
\hline
\end{tabular}

В табл. 2 приведены значения расходов воздуха для приточных и вытяжного отверстий в процентах после уменьшения шага сетки конечных элементов. Значения изменений расходов после второго изменения шага можно считать незначительными и дальнейшие изменения шага сетки не требуются.

Таблица 2

Погрешность в определении расхода воздуха, \%

\begin{tabular}{|l|c|c|}
\hline \multicolumn{1}{|c|}{ Зона } & $\begin{array}{c}\text { Погрешность } \\
\text { после опыта 1 }\end{array}$ & $\begin{array}{c}\text { Погрешность } \\
\text { после опыта 2 }\end{array}$ \\
\hline Приточное отверстие 1 (первый этаж) & 1,2 & 4,2 \\
\hline Приточное отверстие 2 (второй этаж) & 3,0 & 0,1 \\
\hline Приточное отверстие 3 (третий этаж) & 3,5 & 0,7 \\
\hline Вытяжное отверстие & 2,5 & 1,3 \\
\hline
\end{tabular}

В табл. 3 представлен отчет о средней скорости воздуха, полученной в результате расчета для базовой сетки конечных элементов и 7000 итераций (опыт 1), после применения уменьшения шага сетки вдоль границ и дальнейших 3000 итераций (опыт 2) и после применения локального сгущения сетки и последующих 3000 итераций (опыт 3).

Все скорости находятся в допустимом диапазоне. Тем не менее скорость в приточном отверстии на нижнем этаже здания намного больше, чем на верхнем этаже. Следовательно, во избежание возможных сквозняков в рабочей зоне можно рекомендовать запроектировать приточное отверстие внизу большего размера. 
Определение средней скорости воздуха, м/с

\begin{tabular}{|l|c|c|c|}
\hline \multicolumn{1}{|c|}{ Зона } & Опыт 1 & Опыт 2 & Опыт 3 \\
\hline Приточное отверстие 1 (первый этаж) & 0,77 & 0,77 & 0,73 \\
\hline Приточное отверстие 2 (второй этаж) & 0,56 & 0,54 & 0,48 \\
\hline Приточное отверстие 3 (третий этаж) & 0,35 & 0,34 & 0,26 \\
\hline Вытяжное отверстие & 0,71 & 0,69 & 0,64 \\
\hline
\end{tabular}

Суммируя вышеизложенное, можно сказать, что изменение шага сетки значительно влияет на точность значений ключевых параметров микроклимата здания в результатах моделирования. Это становится особенно актуальным для зданий с естественной вентиляцией, так как в этом случае имеет место большое количество зон, представляющих особый интерес, например, приточные и вытяжные отверстия. Приточные отверстия часто располагаются около рабочей зоны, следовательно, очень важно представлять адекватную модель распределения воздушного потока с относительно точными значениями параметров воздуха в целях обеспечения лучших условий комфорта.

Анализ микроклимата здания с использованием программного пакета ANSYS FLUENT позволяет получить более точные результаты расчетов в процессе проектирования, особенно если требуется моделирование процесса.

Результаты моделирования позволили получить выводы об удовлетворительной работе системы естественной вентиляции в здании с атриумом, а также определить скорости воздуха в приточных отверстиях с целью оптимизации их размеров при проектировании здания.

Использование представленной методики может быть дорогим и затратным по времени в случаях необходимости проведения детального анализа. Поэтому следует критически выбирать проект, в котором применение представленной методики будет оправдано и приведет к корректным результатам. Поскольку при проектировании естественной вентиляции процесс моделирования является необходимым, применение пакета ANSYS FLUENT является приемлемым и целесообразным. 


\section{Библиографический список}

1. Directive 2010/31/EU of the European Parliament and of the Council of 19 May 2010 on the energy performance of buildings. - Official Journal of the European Union. 2010. - № 153. - Р. 13-35.

2. Баушева М.Д. Эволюция атриумных пространств: от элемента древней жилищной архитектуры до ключевого элемента в композиции современных гостиниц // Известия КГАСУ. Теория и история архитектуры, реставрация и реконструкция историко-архитектурного наследия. - 2011. - № 4 (18). - С. 16-22.

3. Швалева О.В. Атриумные пространства в структуре жилых зданий [Электронный ресурс] // Известия вузов. Архитектон. - 2010. - № 30. - URL: http://archvuz.ru/2010_22/30.

4. Пикущак Е.В., Миньков Л.Л. Метод расчета аэродинамического коэффициента полезного действия осевого вентилятора // Вестник Томского государственного университета. Математика и механика. - 2016. - № 1(39). - С. 90-101.

5. Pinelli M., Suman A. A numerical method for the efficient design of free opening hoods in industrial and domestic applications // Energy. - 2014. - Vol. 74.

6. Левин Л.Ю., Семин М.А., Газизуллин Р.Р. Разработка метода расчета местных аэродинамических сопротивлений при решении сетевых задач воздухораспределения // Горный журнал. - 2016. - № 3. - С. 200-205.

7. ANSYS FLUENT. Tutorial Guide: Release 14.0. 2011. ANSYS Inc.

8. CIBSE. Environmental Design CIBSE Guide A. The Chartered Institution of Building Services Engineers. - London, 2015. - P. 5-20.

9. British Standards Institution BS EN ISO 13789:2007. Thermal performance of buildings - Transmission and ventilation heat transfer coefficients - Calculation method. London, 2007. - $19 \mathrm{p}$.

10. CIBSE. Heating, ventilating, air conditioning and refrigeration CIBSE Guide B. The Chartered Institution of Building Services Engineers. - London, 2005. - P. 2-16.

11. Ferziger J.H., Peric M. Computational Methods for Fluid Dynamics. - 3rd rev. ed. - Berlin, Heidelberg, New York, Barcelona, Hong Kong, London, Milan, Paris, Tokyo: Springer, 2002. - P. 71-89.

12. Etheridge D. Natural ventilation of buildings theory, measurement and design. Chichester: John Wiley \& Sons, Ltd, 2012. - 157 p.

13. Passive House Institute. Criteria for the Passive House, EnerPHit and PHI Low Energy Building Standard. 2015. - URL: http://www.passiv.de/downloads/03_building_ criteria_en.pdf (accessed 29 April 2016).

14. State of the Art Review - Methods and Tools for Designing Integrated building concepts / I. Andresen, T. Kleiven, M.-A. Knudstrup, P. Heiselberg. - Aalborg, 2008. $-65 \mathrm{p}$.

\section{References}

1. Directive 2010/31/EU of the European Parliament and of the Council of 19 May 2010 on the energy performance of buildings (eds). Official Journal of the European Union. 2010, OJ L 153, pp. 13-35. 
2. Bausheva M.D. Evolutsiya atriumnykh prostranstv: ot elementa drevnej zhilishchnoj arkhitektury do klyuchevogo elementa v kompozitsii sovremennikh gostinits. Izvestiya KGASU. Teoriya i istoriya arkhitektury, restavratsiya i rekonstruktsiya istorikoarkhitekturnogo naslediya, 2011, no. 4 (18), pp. 16-22.

3. Shvaleva O.V. Atriumnye prostranstva v strukture zhilykh zdanij. Izvestiya vuzov. Arkhitekton, 2010, no. 30, available at: http://archvuz.ru/2010_22/30.

4. Pikushak E.V., Minkov L.L. Metod rascheta aerodinamicheskogo koeffitsienta poleznogo dejstviya osevogo ventilyatora. Vestnik Tomskogo gosudarstvennogo universiteta. Matematika i mekhanika, 2016, no. 1 (39), pp. 90-101.

5. Pinelli M., Suman A. A numerical method for the efficient design of free opening hoods in industrial and domestic applications. Energy, 2014, vol. 74.

6. Levin L.Yu., Semin M.A., Gazizullin R.R. Razrabotka metoda rascheta aerodinamicheskikh soprotivlenij pri reshenii setevikh zadach vozdukhoraspredeleniya. Gornyi jurnal, 2016, no. 3, pp. 200-205.

7. ANSYS FLUENT. Tutorial Guide: Release 14.0. 2011. ANSYS Inc.

8. CIBSE. Environmental Design CIBSE Guide A. The Chartered Institution of Building Services Engineers. London, 2015, pp. 5-20.

9. British Standards Institution BS EN ISO 13789:2007. Thermal performance of buildings - Transmission and ventilation heat transfer coefficients - Calculation method. London, 2007. 19 p.

10. CIBSE. Heating, ventilating, air conditioning and refrigeration CIBSE Guide B. The Chartered Institution of Building Services Engineers. London, 2005, pp. 2-16.

11. Ferziger J.H., Peric M. Computational Methods for Fluid Dynamics. Berlin, Heidelberg, New York, Barcelona, Hong Kong, London, Milan, Paris, Tokyo: Springer, 2002, pp. 71-89.

12. Etheridge D. Natural ventilation of buildings theory, measurement and design. Chichester: John Wiley \& Sons, Ltd, 2012. 157 p.

13. Passive House Institute. Criteria for the Passive House, EnerPHit and PHI Low Energy Building Standard, 2015, available at: http://www.passiv.de/downloads/03 building_criteria_en.pdf (accessed 29 April 2016).

14. Andresen I., Kleiven T., Knudstrup M.-A., Heiselberg P. State of the Art Review Methods and Tools for Designing Integrated building concepts. Aalborg, 2008. 65 p.

\section{Получено 24.10.2016}




\section{T. Krasheninnikova, A. Grishkova}

\section{ANALYSIS OF THERMAL ENVIRONMENT \\ IN THE ATRIUM BUILDING UNDER GIVEN CONDITIONS USING THE ANSYS FLUENT SOFTWARE}

Energy efficiency and environmental safety are criteria gaining the most significance in the design and construction processes of modern buildings. The combination of engineering and architectural solutions can provide greater results in meeting these criteria, and an example of such an approach is atrium buildings. The presence of an atrium can reduce electrical energy consumption due to the increase in natural light and reduction in building heat losses. At the same time the atrium increases the bouncy effect which enables more sustainable natural ventilation instead of mechanical one, so removing the energy requirement.

This paper investigates the feasibility of using the ANSYS FLYENT software to model the natural ventilation system's performance in atrium buildings. The general methodology principles used in Great Britain were implemented for the thermal environment assessment, and the assessment of the simulation results has been done based on the extracted plotting materials. An atrium building where the top of the atrium is $6 \mathrm{~m}$ above the roof is proposed as a subject for investigation. The calculations have been provided for the summer period with an external air temperature of $23.4{ }^{\circ} \mathrm{C}$. The results for mass flow rate of fresh and exhaust air along with inlets and outlet air velocity have been provided. The feasibility of such investigations for natural ventilation system's performance along with their modelling and efficiency of the considered systems was assessed.

Keywords: natural ventilation, ventilation of atrium buildings, ANSYS FLUENT software, Finite Volume Method.

Крашенинникова Татьяна Сергеевна (Ноттингел, Великобритания) - магистрант кафедры архитектуры и зеленого строительства университета Hоттингела, e-mail: simastf@rambler.ru.

Гришкова Алла Викторовна (Перль, Россия) - канд. техн. наук, доцент кафедры теплогазоснабжения, вентиляиии и водоснабжения, водоотведения, Перлский национальный исследовательский политехнический университет, e-mail: alla-grishkova@yandex.ru.

Krasheninnikova Tatyana (Nottingem, UK) - Master student, Department of Architecture and Built Environment at the University of Nottingham,e-mail:simastf@rambler.ru.

Grishkova Alla (Perm, Russian Federation) - Ph.D. in Technical Sciences, Associate Professor, Department of Heat and Gas Supply, Ventilation, Water Supply and Sewerage, e-mail: alla-grishkova@yandex.ru. 